\title{
Impact of Pager Notification on Report Verification Times ${ }^{1}$
}

Kader Karli Oguz, MD, David M. Yousem, MD, Tom Deluca, Edward H. Herskovits, MD, PhD Norman J. Beauchamp, Jr, MD, MHS

\begin{abstract}
Rationale and Objectives. The purpose of this study was to assess the impact on times of verification (TOVs) by a pager notification system (PNS) that informs physicians when reports are available for signature.

Materials and Methods. An automated PNS was implemented in the authors' department in November 2000. Monthly report verification times of each physician were collected for 3 months in the years before and after initiation of the PNS. Radiologists enrolled in the PNS and those who were not were assigned into two groups for analysis. Mean TOVs for the two sets of 3 months and for the two groups were calculated and differences recorded. Two-tailed $t$ tests were used to assess for statistical differences between the groups.
\end{abstract}

Results. Twenty-nine of 37 radiologists voluntarily enrolled in the PNS (group 1). Mean TOV was 26.75 hours (standard deviation $[\mathrm{SD}]=17.76)$ for these physicians before and 14.48 hours $(\mathrm{SD}=11.86)$ after the PNS was employed $(P<$ $.01)$. For those physicians who did not enroll in the PNS, mean TOV was 11.53 hours $(\mathrm{SD}=5.55)$ before and 9.77 hours $(\mathrm{SD}=9.86)$ after the PNS was employed $(P=.33)$. Both the absolute and percentage reductions in TOVs were significantly greater for those physicians enrolled in the PNS than for those who were not $(P=.035)$. Twenty-three of 29 (79\%) physicians who used the PNS showed a reduction in their report turnaround times.

Conclusion. Linking the PNS with the radiology information system to notify physicians of unsigned reports was effective in reducing report verification times.

Key Words. Clinical operations, radiology reporting, economics.

๑ AUR, 2002

Improving turnaround times for radiology reports has been one of the major concerns in a practice. In addition to improving communication between physicians by producing an official report more rapidly, a reduction in turnaround times can also produce increased revenue because of the fewer days during which charges are in accounts receivable (1-8). Report turnaround times are also one of the standards regulated by the American College of Radi-

\section{Acad Radiol 2002; 9:954-959}

${ }^{1}$ From the Russell H. Morgan Department of Radiology and Radiological Sciences, Division of Neuroradiology, Johns Hopkins Hospital, 600 N Wolfe St, Phipps B-112, Baltimore, MD 21287. Received April 18, 2002; accepted April 29. K.K.O. supported in part by TUBITAK (Scientific and Technical Research Council of Turkey). Address correspondence to D.M.Y.

(c) AUR, 2002 ology (Standard for Communication-Diagnostic Radiology), accreditation organizations, utilization review panels, and some managed care organizations. From a medicolegal standpoint, a single untoward event resulting from a delay in transcription and report verification could lead to a monetary settlement that far outweighs the cost of extra transcriptionists or information technology solutions.

As some departments have evolved from paper production of radiology reports to a complete electronic patient record, use of electronic signatures in radiology reports has become common. Previously, transcribed paper reports could be placed at the radiologist's location for immediate signature after a transcriptionist concluded a parcel of work. Whether those reports actually were signed immediately, were edited and retyped, or were left for 
review at another time varied from instance to instance. With electronic radiology reports, however, notification that the report is available for finalization has been increasingly left to the direction of the information technology group in the radiology department charged with creating an appropriate warning system. In many cases, this notification may require the radiologist to be logged into the radiology information system (RIS) to receive that notification. This requirement leads to further delays in report verification times.

Verification of reports is but one element of overall report turnaround times, but it constitutes $36 \%$ (mean, 10.6 hours of 29.3 mean total hours) of the overall process time in our department as of the first quarter of 2002. We recently adopted a pager notification system (PNS) that periodically scans the RIS and notifies radiologists when they have reports awaiting their signature. The purpose of this study was to assess the impact on times of verification (TOVs) by the PNS.

\section{MATERIALS AND METHODS}

Because of a perceived need for improvement in report verification times, the manager (T.D.) of the information technology unit in the Russell H. Morgan Department of Radiology and Radiological Sciences (Johns Hopkins Hospital, Baltimore, Md) was consulted about reducing TOVs. A system for notifying physicians of reports awaiting electronic signature was implemented in November 2000. This system used an automated paging system (discussed later) that alerts the radiologists to reports awaiting their signature.

Three times a day on weekdays (8:00 AM, 12:00 noon, and 8:00 PM) and twice a day on weekends (12:00 noon and 8:00 PM), a scheduled query for unsigned reports runs on the RIS (SMS version 22; Siemens, Erlangen, Germany). These data are sent by means of file transfer protocol to a UNIX host. This batch report includes the email address of each radiologist's alphanumeric pager. A short message is coded with the number of unsigned reports for that radiologist. On the UNIX host, a UNIX Task Scheduler (CRONTAB) job runs a UNIX Stream Editor script that strips the digital virtual memory system line terminations from the RIS and then parses out the e-mail address from the rest of the message. This is piped to the UNIX restricted mail function and e-mailed to the radiologist's pager as a text message. A verification message to indicate the CRONTAB job has been completed is then sent to the RIS administrator.
Monthly report verification times for each clinical faculty member in our department were collected for the same time period (July, August, and September) of the years 2000 and 2001, which corresponds to months before and after initiation of the PNS. Only those faculty members who were present for both years and who had verifiable data were included in the study. Radiologists who were placed on the PNS and those who were not were assigned into two groups for analysis. Average TOVs for the 3 months were calculated, and the difference in TOVs between the 2 years was recorded (TOV in 2001 minus TOV in 2000) for each radiologist. Twotailed $t$ tests were used to assess for statistical differences between the two groups.

Because we identified a wide difference between the baseline TOVs of the one group and the baseline TOVs of the other group, we also analyzed the percentage difference between the years 2001 and 2000 in our analysis. The differential change between the 2 years for each group was also assessed for statistical difference to eliminate the impact of any extraneous factors within the department that might affect the overall faculty TOVs in a systemic fashion.

Subsequent analysis separated the members of the division of neuroradiology, who use a voice-recognition system for reporting more than $80 \%$ of their studies, from the members of the division of interventional radiology, who use transcriptionists for more than $80 \%$ of their studies. The impact of the PNS was compared in these two groups, both of which were enrolled completely in the PNS.

\section{RESULTS}

Twenty-nine of 37 radiologists voluntarily enrolled for pager notification after November 2000 (group 1). All physicians were offered the opportunity to join the PNS system, but no systemic biases were identified regarding who enrolled and who did not. The mean TOV was 26.75 hours (standard deviation $[\mathrm{SD}]=17.76$ ) for the 29 physicians who opted for the PNS system before (year 2000) the PNS was initiated and 14.48 hours $(\mathrm{SD}=11.86)$ after (year 2001) the PNS was employed. This difference between the 2 years in subjects using the PNS was statistically significant $(P<.01)$. The difference constituted a $45.9 \%([26.75-14.48] / 26.75)$ reduction in TOVs for the intervention group.

For those eight physicians who did not enroll in the PNS, the mean TOV was 11.53 hours $(\mathrm{SD}=5.55)$ for 
the months before implementation of the system (2000) and 9.77 hours $(\mathrm{SD}=9.86)$ for the months afterward (2001). This difference between the 2 years was not statistically significant $(P=.33)$. The percentage drop in the nonintervention group was $15.3 \%$ ([11.53 - 9.77]/11.53). The percentage drop in TOVs for the PNS group was significantly different $(P<.001)$, but it was not significantly different for the control group $(P=.623)$. By analyzing the percentage drops instead of the absolute drops, we could address the issue of differences in the baseline TOVs between the groups.

Because both groups showed a reduction in TOVs during the study period, we analyzed the data for the difference in the decreases. In this way, we could control for any overall departmental influences that may have occurred during the study period that might have contributed to those reductions. The improvement in TOVs in terms of the difference in hours was statistically significantly different between the two groups $(P=.035)$, with a greater reduction observed for the physicians who enrolled in the PNS (mean, 12.27 hours reduction and $45.9 \%$ for PNS physicians vs 1.76 hours and $15.3 \%$ for non-PNS physicians). This shows a significant difference in the TOV reductions. The sample sizes of these two groups were different, but all the statistical tests cited to this point achieved statistical significance, even with these sample sizes, for the PNS versus non-PNS group comparisons.

Twenty-three of 29 physicians (79\%) who used the PNS showed a reduction in their report turnaround times. Five of eight radiologists (62\%) who were not in the PNS system also showed decreased report verification times in 2001 compared with the same 3-month period in 2000.

We sought to determine the impact of the reporting mechanism on the PNS intervention. When the data were culled for the neuroradiologists $(n=9)$ who used a voice-recognition dictation system for their reports, a reduction in report verification times from 16.42 to 8.35 hours $(P=.015)$ was observed. For interventional radiologists $(n=6)$ who used only a transcription service, the reduction in TOV was even greater, from 61.05 to 14.79 hours $(P=.009)$. We report these data to suggest that differences were obtained despite variable means of inputting the dictations (ie, transcriptionists vs automated voice input).

The impact on the overall departmental report turnaround times, which includes the time from completion of study to the finalized report, from September 2000 to September 2001 was positive (from 46.54 to 31.63 hours). This represented a reduction of 14.91 hours or $32 \%$ ([46.54 - 31.63]/46.54).

\section{DISCUSSION}

Because a finalized radiology report is the major form of communication between the radiologist and the clinician regarding patient care, prompt delivery of this report is one of the standards by which radiology departments are assessed. External forces such as the Joint Commission on the Accreditation of Health Care Organizations also survey turnaround times as a standard of quality assurance.

A typical flow chart of the report turnaround process includes $(a)$ performance of the study, $(b)$ registration of the data for study completion, $(c)$ delivery of the images to the radiologist, $(d)$ dictation of the study, $(e)$ transcription of the dictation, and $(f)$ verification of the radiology report. We do not list the "delivery of the report" step, because with an electronic patient record, printing or delivering the report may be automated through e-mail or autofaxing. In either case, however, the report is readily available for review. A delay in any one of these steps results in increased report turnaround times. Whereas workflow issues and transcriptionist availability affect the first steps in the process, the radiologist is primarily responsible for the last step (between the time of transcription and the TOV). The American College of Radiology Standard for Communication indicates that the final report should be proofread to minimize typographic errors, deleted words, and confusing or conflicting statements (9). Studies to date have focused on the effect of picture archiving and communication systems (PACS), workstations, and voice-recognition systems on report turnaround times, but, to our knowledge, little work has been performed regarding the TOV, which often depends solely on the radiologist (10). A verified report is the only official document that clinicians can use to manage a patient's case. By integrating the report into the electronic patient record as early as possible, more expeditious treatment of patients may ensue.

The advent of digital image generation and communication technology is causing changes in radiology workflow and helping to improve turnaround times. With digital technology, the time between performance of imaging and delivery of an image to the radiology reader is reduced to a matter of seconds or, at most, minutes. Because a PACS eliminates workload by reducing the number of hard-copy films produced (also saving money), is 
as reliable as a hard-copy environment, and decreases the number of lost film images, it has found a high acceptance rate in radiology departments $(5,11,12)$. Several studies $(1,5,11,13-17)$ have shown an apparent reduction in the time of image delivery to the radiologist, retrieval of images, interpretation, and total report turnaround with use of a PACS. Integration of a PACS into the hospital information system and RIS has an additional positive impact on report turnaround time and communication between physicians (18-20). On the other hand, Mehta et al (21) found no difference in time to final reports being signed by staff radiologists, but they did find an $85 \%$ improvement in time to a preliminary report by a resident or fellow when a PACS system was employed. These authors attributed the results to a relatively persistent, lackadaisical attitude toward signing on the part of staff radiologists. We sought to address this problem through implementation of our PNS system.

Computer-based voice-recognition dictation systems have now placed the radiologist beyond mere dictation and into the transcription process in addition to the end stage of report verification. The time required for dictation using a voice-recognition system is longer than that required using a tape-based transcription system, but the lack of intermediary involvement by a transcriptionist and the capability of immediate final approval of the report by the staff radiologist makes the total turnaround time shorter with a voice-recognition system $(7,22,23)$. If the workflow is designed such that the primary reader of the film images is immediately signing off on his or her interpretations rather than "batching" them to be signed later, TOVs and, hence, report turnaround times can be reduced by an even greater margin. Voice-recognition systems confer extra time for reporting by a factor of 97.5 additional seconds per plain film (from 41.9 seconds with a tape system to 139.4 seconds with a voice-recognition system) (24). If one has a busy practice, this can lead to hours of additional work time by the radiologist. Unless immediate signature is a feature of the system, issues with report verification might still remain.

Alternative methods of immediate reporting and verifying of reports (eg, manual coding with SCRIBE or RAPORT) can also be employed. With SCRIBE, radiologic findings and anatomic terms are presented in a graphic design, and reporting is directed with a trackball or a touch-sensitive video screen (25). These alternative methods have effectively reduced turnaround times from 8 hours to 15 minutes and from 36 hours to 4 hours (25). Such reporting systems have been criticized, however, because of their telegraphic speech or lack of clarity (25). Even with those programs in which voice-recognition dictation systems are used, a trainee usually produces a preliminary report that is subsequently signed by a faculty member, which incurs a natural delay in TOV.

Delays in report verification occur for many reasons: (a) unavailability of radiologists on evenings, weekends, and vacations, $(b)$ necessity for double signatures (by a trainee and a staff member), (c) lack of free time during the workday to sign reports, $(d)$ lack of available access terminals, $(e)$ low motivation, $(f)$ large volumes of reports that require careful scrutiny, $(g)$ limited direct rewards for signing cases promptly, $(h)$ lack of communication and synchrony in schedules between the transcriptionist and radiologist, and (i) lack of awareness that reports are even available for signature (26).

Our department has no double-signing rule, and proxy signing is forbidden. We also have adequate terminals dispersed throughout the hospital. Signing reports by using personal computers at home and in the office also is possible for members of our department. More recently, report verification times have been added to the criteria used for calculating bonuses. Nonetheless, before instituting the PNS program, we remained subject to a lack of awareness that cases were pending for signature.

Seltzer et al (26) used techniques of total quality management to speed radiologists in signing reports. The interventions they enacted included installing home computer terminals and a buddy system for proxy signing, eliminating the requirement for a trainee signature (ie, double signature), adding more computer terminals, improving radiologist motivation, teaming groups of radiologists with specific transcriptionists, and streamlining the transcription service. These authors found that the mean time required to sign reports decreased by 59\% (from an initial value of 26.0 hours [SD $=8.4$ hours] to 10.6 hours $[\mathrm{SD}=2.9$ hours $]$ ) through use of these steps (26). The gains were hard to maintain, however; within 4 months after the pilot study, $20 \%$ of the benefit had been lost. This pilot study in one departmental section of a teaching hospital was later extended and found to be feasible for use by the entire radiology department (27). The same interventions used in the pilot study, plus the use of bar codes for standardized reports and more prompt delivery of film images to the radiologists, yielded an overall improvement in report verification times of $68 \%$ (from a mean of 41.3 hours to 13.3 hours) (27).

The PNS employed at our hospital requires an interface between the RIS and the pager network. The infor- 
mation system can automatically search for individuals with outstanding reports, and it can generate a text message on the display on the physician's beeper. This text display reminds the physician of the outstanding reports and indicates how many are overdue. This is a relatively innocuous system that serves as a reminder and does not require increased personnel to maintain.

The data analysis in our study was designed to look at three factors: $(a)$ whether the PNS effected a change in verification times, $(b)$ whether any changes in verification times resulted from other, extraneous factors occurring at the same time (hence, we looked for statistical differences in the degree of change between the two groups during the two time periods), and $(c)$ whether any gains observed were specific to those individuals who report their cases by using voice-recognition software versus traditional dictation and transcription. To that end, we compared preand post-PNS verification times in the same individuals and found statistically significant absolute and percentage reductions in verification times (from a mean of 26.75 to 14.48 hours $[P<.01$ and $P<.001]$, or a percentage decrease of more than $45 \%$ ). We also showed that, during the same time frame, those individuals who did not subscribe to the PNS did not show a statistically significant change (reduction of 1.76 hours and $15.3 \%, P=.334$ and .623 , respectively). That no extraneous factors in the department affected the results was further supported by the differences between the 2000 and 2001 times of the two groups being significantly different $(P=.035)$; that is, radiologists notified by pagers had, on average, a greater reduction in verification time than did radiologists not notified by pagers when comparing 2000 and 2001 data. Finally, we took a group of radiologists who used voicerecognition software to transcribe their reports (neuroradiology division) and a group of radiologists who used human transcriptionists exclusively (body interventionalists) and showed that both groups had a statistically significant reduction in report verification times after employing the PNS ( $P=.015$ and .009 , respectively). This demonstrated the impact of the PNS on two methods of inputting reports.

Other potential causes of systematic biases that may not be evident in this retrospective analysis could have influenced the data. No gross changes in departmental policies were enacted during the period of study, and we could not directly identify a bias regarding who volunteered for the PNS system versus who did not. Nonetheless, it would appear from the baseline data that the individuals with the longest preintervention TOVs tended to join the PNS group. Perhaps they were more highly motivated to effect changes in their TOVs. Therefore, we analyzed the differences between the two groups as well as the absolute reductions. The $100 \%$ enrollment of the neuroradiology and body interventional groups as opposed to the diagnostic radiology groups may also reflect a bias toward the special-procedure specialties, the motivations of their divisional leaders, or both.

The eight physicians in the non-PNS group, both before and after the intervention, had a shorter mean TOV than those using the PNS. This may be because of a preponderance of individuals who used the SCRIBE automated report system (25), which leads to shorter verification times similar to those obtained with use of voicerecognition systems. Therefore, we also examined the percentage of decrease and the absolute decrease in TOVs, and we found that the PNS group had a significantly greater percentage decrease in TOV. We also looked at a subgroup using voice-recognition automated systems (neuroradiology division) and found that this group still showed a significant drop in TOVs after employing the PNS. It may be that the non-PNS individuals were sufficiently self-motivated that they were able to keep their TOVs reasonable even without the prompting of a pager system, or perhaps they had already adopted a system of periodic checks of the RIS for unsigned reports. In any case, there remained a dramatic reduction, by more than 12 hours in TOVs for the 29 individuals and by nearly 48 hours for the interventional radiology team, that greatly benefited the department.

Verification times may be seasonal in their length. In part, this may result from vacations planned during the summertime, annual meetings such as that of the Radiological Society of North America, and holidays (eg, Christmas or Easter). In part, it may also result from the nature of the change in trainees during the month of July, when review of the reports may be slower as the trainees familiarize themselves with the hospital system. One can often see spikes and valleys in the verification times when plotted over a year-long course. Therefore, we were careful to analyze the same 3-month periods during 2000 and 2001, both before and after the PNS was employed. Thus, time of year was a controlled variable.

Our results are in agreement with those of a study in which implementation in an emergency department of a similar automated paging system for notification of image availability provided more timely reporting and less lost cases without a report (8). 
In our department, the PNS system was effective in reducing the report verification time by, on average, more than 12 hours for physicians using it, which could be translated to better service and reduced time in accounts receivable. Reductions in verification times also improve patient care, because referring physicians can access finalized reports more quickly. This may lead to reduced lengths of hospital stays, which could also positively influence a hospital's finances. The "cost" of implementing the PNS was the cost of converting analog pagers to digital ones and of the programming time for the information technology specialist.

The TOV is only one component of the overall report verification time, but it has a direct, positive impact (contributing to overall reductions in our departmental turnaround times by more than $32 \%$ ). This simple intervention can have substantial positive impact on report processing times. Subsequent to this study, a private practice group of more than 70 radiologists affiliated with our department implemented the system on a different PACS/ RIS system and has also shown beneficial results (oral communication, January 2002).

In summary, linking the PNS with the RIS to notify physicians of unsigned reports is a worthwhile investment for reduction in TOV and, thereby, overall report turnaround time, which is one important component to quality improvement efforts.

\section{REFERENCES}

1. Redfern RO, Horii SC, Feingold E, Kundel HL. Radiology workflow and patient volume: effect of picture archiving and communication systems on technologists and radiologists. J Digit Imaging 2000; 13(suppl 1): 97-100.

2. Huang HK, Lou SL, Dillon WP. Neuroradiology workstation reading in an interhospital environment: a 19-month study. Comput Med Imaging Graph 1997; 21:309-317.

3. Passadore DJ, Isoardi RA, Ariza PP, Padin C. Use of a low-cost, PCbased image review workstation at a radiology department. J Digit Imaging 2001; 14(suppl 1):222-223.

4. Tamm EP, Raval B, West OC, Dinwiddie S, Holmes R. Evaluating the impact of workstation usage on radiology report times in the initial 6 months following installation. J Digit Imaging 1999; 12(suppl 1):152154.

5. Twair AA, Torreggiani WC, Mahmud SM, Ramesh N, Hogan B. Significant savings in radiologic report turnaround time after implementation of a complete picture archiving and communication system (PACS). J Digit Imaging 2000; 13:175-177.

6. Sinha U, Dai B, Johnson DB, et al. Interactive software for generation and visualization of structured findings in radiology reports. AJR Am J Roentgenol 2000; 175:609-612.
7. Hundt W, Stark O, Scharnberg B, et al. Speech processing in radiology. Eur Radiol 1999; 9:1451-1456.

8. Andriole KP, Avrin DE, Weber E, Luth DM, Bazzill TM. Automated examination notification of emergency department images in a picture archiving and communication system. J Digit Imaging 2001; 14(suppl 1):143-144.

9. American College of Radiology. ACR standard for communication: diagnostic radiology. In: Standards. Reston, Va: American College of Radiology, 2001; 1-31.

10. Beard DV, Hemminger BM, Pisano ED, et al. Computed tomography interpretations with a low-cost workstation: a timing study. J Digit Imaging 1994; 7:133-139.

11. Mattern CW, King BF Jr, Hangiandreou NJ, et al. Electronic imaging impact on image report turnaround times. J Digit Imaging 1999; 12(suppl 1):155-159.

12. Kundel HL, Polansky M, Dalinka MK, et al. Reliability of soft-copy versus hard-copy interpretation of emergency department radiographs: a prototype study. AJR Am J Roentgenol 2001; 177:525-528.

13. Mullins ME, Mehta A, Patel H, McLoud TC, Novelline RA. Impact of PACS on the education of radiology residents' perspective. Acad Radiol 2001; 8:67-73.

14. Reiner BI, Siegel EL, Hooper FJ, Pomerantz S, Dahlke A, Rallis D. Radiologists' productivity in the interpretation of CT scans: a comparison of PACS with conventional film. AJR Am J Roentgenol 2001; 176:861864.

15. Horii SC, Levine BA, Goger G, et al. A comparison of case retrieval times: film versus picture archiving and communication systems. J Digit Imaging 1992; 5:138-143.

16. Bryan S, Weatherburn G, Watkins J, et al. Radiology report times: impact of picture archiving and communication systems. AJR Am J Roentgenol 1998; 170:1153-1159.

17. Redfern RO, Kundel HL, Polansky M, Langlotz CP, Horii SC, Lanken $\mathrm{PN}$. A picture archival and communication system shortens delays in obtaining radiographic information in a medical intensive care unit. Crit Care Med 2000; 28:1231-1232.

18. Inamura $\mathrm{K}$, Umeda $\mathrm{T}$, Harauchi $\mathrm{H}$, et al. Time and flow study results before and after installation of a hospital information system and radiology information system and before clinical use of a picture archiving and communication system. J Digit Imaging 1997; 10:1-9.

19. Strickland NH. Review article: some cost-benefit considerations for PACS —a radiological perspective. Br J Radiol 1996; 69:1089-1098.

20. Honeyman JC. Information systems integration in radiology. J Digit Imaging 1999; 12(suppl 1):218-222.

21. Mehta A, Dreyer K, Boland G, Frank M. Do picture archiving and communication systems improve radiology report turnaround times? J Digit Imaging 2000; 13(suppl 1):105-107.

22. Gale B, Safriel Y, Lukban A, Kalowitz J, Fleischer J, Gordon D. Radiology report production times: voice recognition vs. transcription. Radiol Manage 2001: 23:23-25.

23. Ramaswamy MR, Chaljub G, Esch O, Fanning DD, van Sonnenberg E. Continuous speech recognition in MR imaging reporting: advantages, disadvantages, and impact. AJR Am J Roentgenol 2000; 174:617622.

24. Seltzer RA, Reimer GW, Cooperman LR, Rossiter SB. Computerized radiographic reporting in a community hospital: a consumer's report. AJR Am J Roentgenol 1977; 128:825-829.

25. Bluemke DA, Eng J. An automated radiology reporting system that uses HyperCard. AJR Am J Roentgenol 1993; 160:185-187.

26. Seltzer SE, Kelly P, Adams DF, et al. Expediting the turnaround of radiology reports: use of total quality management to facilitate radiologists' report signing. AJR Am J Roentgenol 1994; 162:775-781.

27. Seltzer S, Kelly P, Adams DF, et al. Expediting the turnaround of radiology reports in a teaching hospital setting. AJR Am J Roentgenol 1997; 168:889-893. 